\title{
Spinal Anesthesia for Cesarean Delivery in a Woman with Neuromyelitis Optica
}

\author{
Nathaniel Greene, MD, Emily Dinges, MD, Christopher Ciliberto, MD, \\ Margaret Sedensky, MD, PhD, and Ruth Landau, MD
}

\begin{abstract}
Neuromyelitis optica (NMO), or Devic's disease, is an idiopathic severe demyelinating disease that preferentially affects the optic nerve and spinal cord. Neuraxial anesthesia in women with multiple sclerosis is widely accepted, but reports of the use of neuraxial anesthesia in patients with NMO are scarce. We report the case of a morbidly obese primigravida undergoing a planned cesarean delivery at 32 weeks' gestation due to an acute exacerbation of NMO, managed with spinal anesthesia. Other than increased intraoperative hyperalgesia requiring inhaled nitrous oxide/oxygen, the mother experienced no apparent anesthetic-related complications. (A\&A Case Reports. 2014;2:108-10.)
\end{abstract}

$\mathrm{N}$ euromyelitis optica (NMO), or Devic's disease, is an idiopathic severe demyelinating disease of the central nervous system (CNS) that preferentially affects the optic nerve and spinal cord. It has long been considered a "more severe form" of multiple sclerosis (MS). However, recent evidence suggests that NMO is an entirely separate entity, because an antibody to aquaporin-4, a water channel found on CNS astrocytes, ${ }^{1}$ has been identified as a unique biomarker for the disease.

While provision of labor analgesia ${ }^{2}$ or neuraxial anesthesia for cesarean delivery in women with MS is widely accepted, ${ }^{3}$ the safety of neuraxial anesthesia in NMO has not been established. Spinal anesthesia has even been suggested to cause NMO, although this is highly debated. ${ }^{4,5}$ With new insights into the pathophysiology of NMO, the effects of neuraxial techniques on women with $\mathrm{NMO}$ are of increased interest because more women of childbearing age are likely to be diagnosed with this disease in the future.

We report the anesthetic care of a morbidly obese primigravida woman, managed with a spinal anesthetic, requiring a cesarean delivery at 32 weeks due to an acute exacerbation of NMO.

The patient's informed consent was obtained for case report publication.

\section{CASE DESCRIPTION}

A 22-year-old G1P0 woman of mixed Asian and African American origin with a 3-year history of NMO presented at 13 weeks' gestation. She was diagnosed with NMO in 2009 when she presented with blindness and lower extremity weakness. On initial workup, she was found to have optic neuritis, transverse myelitis, and met all supportive criteria for NMO (Table 1). Magnetic resonance imaging (MRI) at

From the Department of Anesthesiology and Pain Medicine, University of Washington, Seattle, Washington.

Accepted for publication October 29, 2013.

Funding: None.

The authors declare no conflicts of interest.

Address correspondence to Ruth Landau, MD, Department of Anesthesiology and Pain Medicine, University of Washington, 1959 NE Pacific St., Box 356540, Seattle, WA. Address e-mail to rulandau@uw.edu.

Copyright (C) 2014 International Anesthesia Research Society

DOI: 10.1213 /XAA.0000000000000016 that time (Fig. 1) revealed intramedullary lesions in the cervical and thoracic spinal cord that had resolved with aggressive treatment of her NMO (prednisone and azathioprine), as seen on her 2010 MRI (Fig. 2). Since her 2010 MRI, she had been managed with azathioprine only. In addition, the patient was morbidly obese (body mass index $=45 \mathrm{~kg} / \mathrm{m}^{2}$ ), with poorly controlled type 2 diabetes mellitus.

She was admitted for treatment of flares of NMO at 21, 24, 28, and 29 weeks' gestation. Her symptoms included severe generalized body itching, excruciating allodynia in which she could not tolerate the touch of any clothes including the hospital patient gown, and profound bilateral lower extremity weakness, preventing ambulation. Her first 3 flares were successfully treated with IV methylprednisolone and plasmapheresis. However, on her 4th admission at 29 weeks, symptoms continued to worsen despite IV methylprednisolone, plasma exchanges, IV immunoglobulin, and azathioprine. With no resolution of symptoms at 32 weeks, a decision was made to proceed with a planned cesarean delivery. With additional consultation from the neurologist managing her NMO flares, the anesthesiology team concluded that neuraxial approaches were not contraindicated in this patient, despite her worsening sensory-motor deficits.

After informing the patient of all the risks and complications of a general anesthetic, spinal anesthetic, a combined spinal-epidural, or even continuous spinal anesthesia, the patient agreed with our plan to proceed with spinal anesthesia after understanding the risks and benefits of each approach and also understanding that we may have to emergently convert the spinal anesthetic to a general anesthetic. Two 18-gauge IV catheters (BD Insyte Autogard TM, Becton Dickinson, Sandy, UT) and an arterial catheter

Table 1. Diagnostic Criteria for Neuromyelitis Optica ${ }^{6,7}$

Definitely required criteria

Optic neuritis

Acute myelitis

At least 2 of 3 supportive criteria

Contiguous spinal cord MRI lesion extending over $>3$ vertebral segments

Brain MRI not meeting diagnostic criteria for multiple sclerosis NMO-IgG seropositive status

$\mathrm{NMO}=$ neuromyelitis optica; $\mathrm{MRI}=$ magnetic resonance imaging; IgG = immunoglobulin $\mathrm{G}$. 


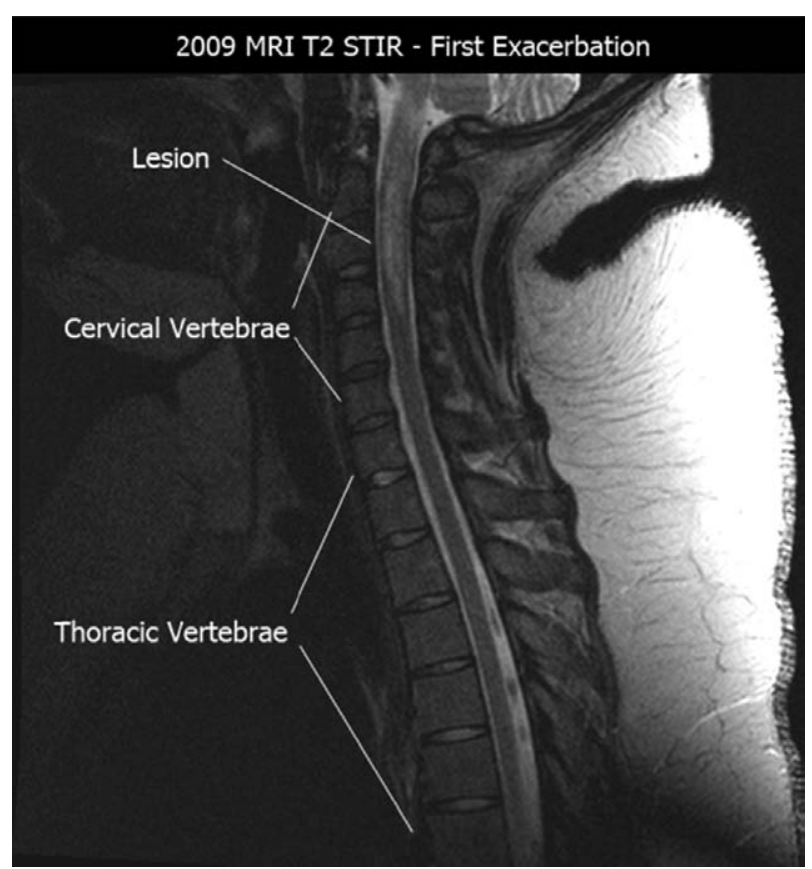

Figure 1. Sagittal spine magnetic resonance image (MRI) T2 with short time inversion recovery (STIR) sequence showing demyelination at $\mathrm{C} 2-3$ at the time of diagnosis of this patient.

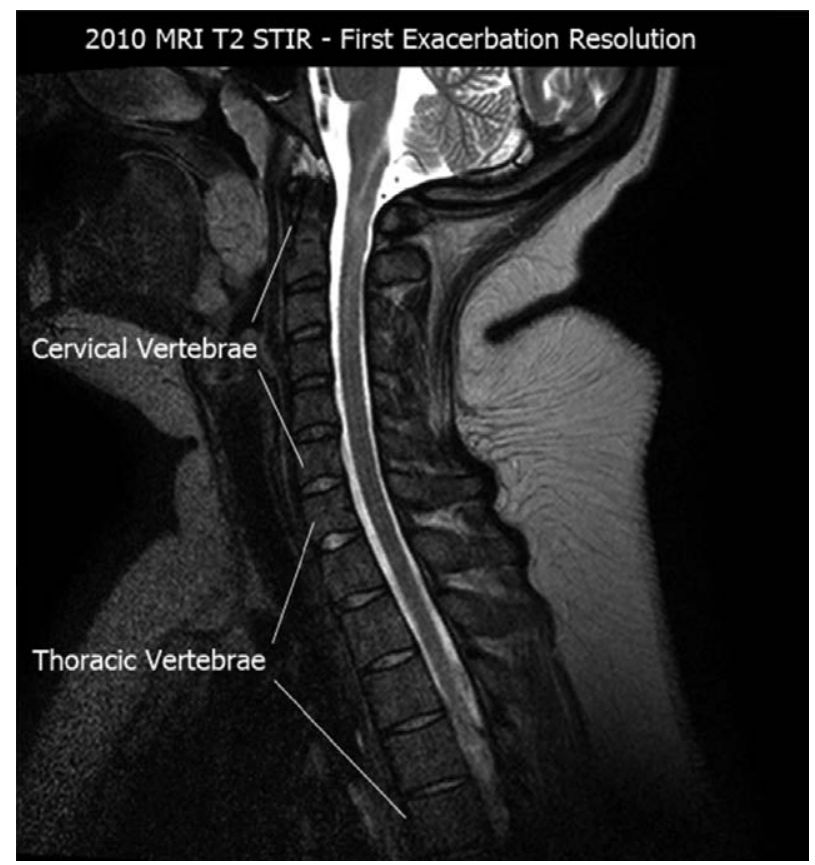

Figure 2. Sagittal spine magnetic resonance image (MRI) T2 with short time inversion recovery (STIR) sequence (2010), showing resolution (no lesions) of her 2009 flare.

(Arrow Quickflash Radial Artery Catherization set, Arrow International, Reading, PA) were inserted. Using a 25-gauge Whitacre needle inserted at a presumed L4-5 level, a solution of hyperbaric bupivacaine $(12 \mathrm{mg})$, fentanyl $(15 \mathrm{mcg})$, and preservative-free morphine $(200 \mathrm{mcg})$ was injected into the subarachnoid space. A phenylephrine infusion was started at $50 \mathrm{mcg} / \mathrm{min}$ at the time of spinal injection. The obstetricians proceeded with the cesarean delivery after a bilateral T4 level of insensibility to pinprick was achieved. While the patient did not discern the initial skin incision and dissection, manipulation of the uterus caused intense pain and burning in the abdominal area. IV fentanyl ( $85 \mathrm{mcg})$, morphine $(2 \mathrm{mg})$, and midazolam $(2 \mathrm{mg})$ were given to alleviate her symptoms, but were completely ineffective. As a last resort before inducing general anesthesia, inhaled 50\% nitrous oxide was administered with complete resolution of burning sensations and all discomfort. An otherwise healthy premature male infant, weighing $1.6 \mathrm{~kg}$ with Apgar scores of 6 and 7 at 1 and 5 minutes, respectively, was delivered. The patient recovered from the spinal anesthetic within 3 to 4 hours. The baby was taken to the neonatal intensive care unit after birth and was discharged home 2 months later. The patient was started on a rituximab infusion 2 days after delivery followed by tapering of the steroid dose.

An MRI performed 72 hours after delivery showed new intramedullary lesions from C3-5 and C7 compared with her previous MRI in 2010, consistent with a demyelinating process (Fig. 3). Neurologic symptoms rapidly resolved over the course of 7 days, and the patient was discharged for rehabilitation 8 days after the delivery.

The patient was contacted 1 year postpartum. She reported no residual neurological deficits other than minor periumbilical numbness. She had recovered her ability to walk and had no allodynia.

\section{DISCUSSION}

Devic first described this disease as a purely monophasic disease with 2 defining symptoms, bilateral visual loss and myelitis. ${ }^{8}$ As these 2 clinical symptoms often presented together, this disease later became known as NMO. NMO is relatively rare with an unknown incidence and prevalence. Specific diagnostic criteria were only established in 2006

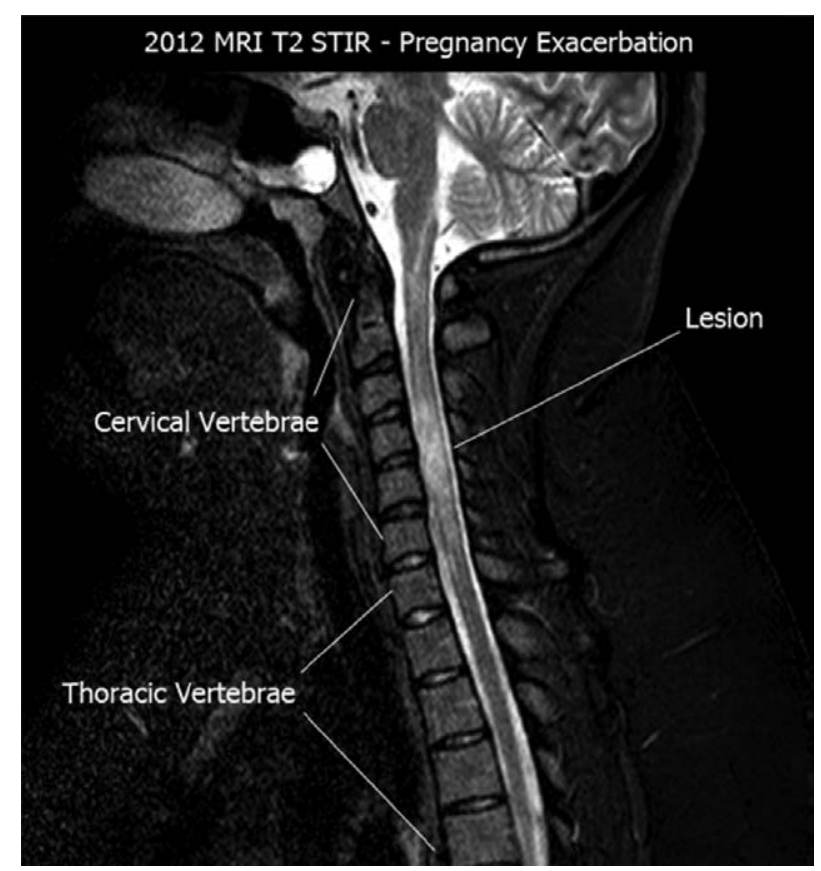

Figure 3. Sagittal spine magnetic resonance image (MRI) T2 with short time inversion recovery (STIR) sequence showing recurrence of the disease during pregnancy in 2012 . 
(Table 1). NMO is likely to represent $<1 \%$ of all CNS demyelinating diseases in the Caucasian population, but $20 \%$ to $48 \%$ of CNS demyelinating disease in certain non-Caucasian populations, particularly in Asia and Africa. ${ }^{9} \mathrm{NMO}$ appears to be more common in women. ${ }^{10}$ The disease has a relapsing form, approximately $80 \%$ of those affected are women. ${ }^{10}$

NMO has long been thought to be a variant form of MS. However, recent studies have shown that while symptoms of the 2 diseases can be similar, their pathophysiologies are substantially different. ${ }^{8}$ Subsequently, the treatments, mostly immunomodulatory, for NMO and MS are distinct. Some treatments that are successful for MS are likely to worsen NMO and the inverse is true as well. ${ }^{10}$ In addition, while MS exacerbations decrease in frequency during pregnancy, ${ }^{11}$ exacerbations of NMO have been shown to increase in frequency in the parturient. ${ }^{12}$

Our patient's symptoms appear to have been exacerbated by pregnancy and relieved after delivery. One of her main complaints was that of severe allodynia in the abdominal area, leading to the decision to perform a preterm scheduled cesarean delivery. After adminstering the spinal anesthetic, despite an adequate bilateral surgical anesthesia to the T4 dermatomal level, and blockade of somatic pain on skin incision, the patient reported severe hyperalgesic visceral pain that resolved completely with nitrous oxide. Nitrous oxide has been shown to prevent opioid tolerance in the perioperative setting, possibly by its $N$-methyl-D-aspartate antagonistic activity. ${ }^{13}$ We can speculate that in the setting of a demyelinating condition such as NMO, there seems to be abnormal peripheral and central sensitization causing chronic pain or abnormal pain sensation such as allodynia (like this patient had), nitrous oxide could then be particularly effective as an analgesic in this disease. Ketamine may have provided the same effect. However, due to the immediate and striking response to nitrous oxide, ketamine was not administered.

There are few published reports describing the use of neuraxial anesthesia in obstetric patients with NMO. Two case reports describe the use of epidural anesthesia in the NMO obstetric population. One describes the management of an urgent cesarean delivery with an epidural anesthetic for a nonreassuring fetal heart tracing at 37 weeks' gestation in a woman previously diagnosed with $\mathrm{NMO}$, with no postoperative complications. ${ }^{14}$ The other reports a patient with NMO who developed temporary neurological symptoms 2 months after an epidural anesthetic causing that patient to elect a general anesthetic for her subsequent delivery, which was done with no postoperative complications. ${ }^{15}$ Two case reports ${ }^{4,5}$ describe development of NMO 1 to 2 days after spinal anesthesia with ensuing permanent neurological deficits, 1 in a parturient who had a cesarean delivery ${ }^{4}$ and 1 in an older woman undergoing orthopedic surgery. ${ }^{5}$ One retrospective cohort study from France using an NMO registry tracked 20 women with a total of 25 pregnancies and found that the course of the disease was similar in women who delivered with epidural analgesia and those who did not. ${ }^{12}$

These various anecdotal reports seem to have examined 2 separate questions. First, is there an association between neuraxial anesthesia in a previously healthy patient and NMO, or in other words, could a spinal anesthetic unmask or precipitate $\mathrm{NMO}$ in an individual who has not yet been diagnosed with the disease? Second, what is the best anesthetic management for a parturient diagnosed with NMO and potentially undergoing surgery or requesting labor analgesia at the time of a flare? The second question is more relevant to our case, and because the major concerns are that neuraxial techniques may cause an exacerbation of $\mathrm{NMO}$, until more spinal anesthetics are delivered in patients with $\mathrm{NMO}$, we cannot conclude that this technique is safe for all patients with NMO.

The impressive response of our patient to the analgesic effect of nitrous oxide was striking, suggesting that nitrous oxide could be an advantageous adjuvant for patients with allodynia in the context of a demyelinating disease. Further reports and outcomes of spinal anesthesia in parturients diagnosed with NMO will be of interest since one can anticipate that more women with this diagnosis will be managed by obstetric anesthesiologists. IH

\section{REFERENCES}

1. Ransohoff RM. Illuminating neuromyelitis optica pathogenesis. Proc Natl Acad Sci U S A 2012;109:1001-2

2. Vukusic $S$, Hutchinson $M$, Hours $M$, Moreau $T$, Cortinovis-Tourniaire $\mathrm{P}$, Adeleine $\mathrm{P}$, Confavreux $\mathrm{C}$, The Pregnancy In Multiple Sclerosis Group; Pregnancy In Multiple Sclerosis Group. Pregnancy and multiple sclerosis (the PRIMS study): clinical predictors of post-partum relapse. Brain 2004;127:1353-60

3. Drake E, Drake M, Bird J, Russell R. Obstetric regional blocks for women with multiple sclerosis: a survey of UK experience. Int J Obstet Anesth 2006;15:115-23

4. Hosseini H, Brugieres P, Degos JD, Cesaro P. Neuromyelitis optica after a spinal anaesthesia with bupivacaine. Mult Scler 2003;9:526-8

5. Facco E, Giorgetti R, Zanette G. Spinal anaesthesia and neuromyelitis optica: cause or coincidence? Eur J Anaesthesiol 2010;27:578-80

6. Krupp LB, Banwell B, Tenembaum S; International Pediatric MS Study Group. Consensus definitions proposed for pediatric multiple sclerosis and related disorders. Neurology 2007;68:S7-12

7. Wingerchuk DM, Lennon VA, Pittock SJ, Lucchinetti CF, Weinshenker BG. Revised diagnostic criteria for neuromyelitis optica. Neurology 2006;66:1485-9

8. Barnett MH, Sutton I. Neuromyelitis optica: not a multiple sclerosis variant. Curr Opin Neurol 2012;25:215-20

9. Wingerchuk DM, Lennon VA, Lucchinetti CF, Pittock SJ, Weinshenker BG. The spectrum of neuromyelitis optica. Lancet Neurol 2007;6:805-15

10. Wingerchuk DM. Neuromyelitis optica. Int MS J 2006;13:42-50

11. Confavreux $C$,Hutchinson M,Hours MM, Cortinovis-Tourniaire $\mathrm{P}$, Moreau T. Rate of pregnancy-related relapse in multiple sclerosis. Pregnancy in Multiple Sclerosis Group. N Engl J Med 1998;339:285-91

12. Bourre B, Marignier R, Zéphir H, Papeix C, Brassat D, Castelnovo G, Collongues N, Vukusic S, Labauge P, Outteryck O, Fontaine B, Vermersch P, Confavreux C, de Seze J; NOMADMUS Study Group. Neuromyelitis optica and pregnancy. Neurology 2012;78:875-9

13. Richebé $P$, Rivat $C$, Creton $C$, Laulin JP, Maurette $P$, Lemaire $M$, Simonnet G. Nitrous oxide revisited: evidence for potent antihyperalgesic properties. Anesthesiology 2005;103:845-54

14. Gunaydin B, Akcali D, Alkan M. Epidural anaesthesia for Caesarean section in a patient with Devic's Syndrome. Anaesthesia 2001;56:565-7

15. Sadana N, Houtchens M, Farber MK. Anesthetic management of a parturient with neuromyelitis optica. Int J Obstet Anesth 2012;21:371-5 